\title{
Drowning by numbers: topology and physics in fluid dynamics
}

\author{
Amaury Mouchet \\ Laboratoire de Mathématiques et de Physique Théorique, Université François Rabelais \\ de Tours, CNRS (UMR 7350), Fédération Denis Poisson, 37200 Tours, France
}

Since its very beginnings, topology has forged strong links with physics and the last Nobel prize in physics, awarded in 2016 to Thouless, Haldane and Kosterlitz "for theoretical discoveries of topological phase transitions and topological phases of matter", confirmed that these connections have been maintained up to contemporary physics. To give some (very) selected illustrations of what is, and still will be, a cross fertilization between topology and physics 1 , hydrodynamics provides a natural domain through the common theme offered by the notion of vortex, relevant both in classical $(\S 2)$ and in quantum fluids $(\S 3)$. Before getting into the details, I will sketch in $\S 1$ a general perspective from which this intertwining between topology and physics can be appreciated: the old dichotomy between discreteness and continuity, first dealing with antithetic thesis, eventually appears to be made of two complementary sides of a single coin.

\section{The arena of the discrete/continuous dialectic}

One century after Thales of Miletus had proposed that water was the natural principle of all things, the first atomists Leucippus and Democritus advocated for a discrete conception of matter. The existence of an ultimate lower limit of divisibility, materialised by the atoms, may have been a logical answer to the Zeno's paradoxes (Stokes 1971, chap. VIII; Bell 2006, chap. I). In some westernmost banks of the Mediterranean sea, the Pythagorean school was concerned by a line of thought following quite an opposite direction: the discovery of the irrational numbers counterbalanced the conception of a universe exclusively driven by the integer and rational - in the original acception of the word-numbers. For twenty-five centuries, the dialectic between continuity and discreteness has never stopped nurturing natural philosophy.

At our daily life scales, the ones for which the brains have been shaped by Darwinian evolution 2, discreteness appears to be an inevitable way for intelligence to model the world 3 . Furthermore, operationally speaking, any measurement is reduced, in the last

\footnotetext{
${ }^{1} \mathrm{~A}$ more general review is proposed by Nash (1999) and a systematic presentation on the topological concepts used by physicists can be found in (Nakahara, 1990).

${ }^{2}$ In modern times physics and chemistry were not, by far, the only scientific disciplines to be shaken by violent debates between discrete and continuous schools; in the xixpcentury Lyell's uniformitarianism in geology, by contrast with catastrophism, had an important influence on the young Darwin. By the way, one can notice that the binary opposition between discreteness and continuity provides by itself a meta self-referring epistemological dichotomy, so to speak.

${ }^{3}$ However, neurology shows that numerical cognition is more analogical than numerical: beyond few units, the numbers are encoded and treated by the brain as fuzzy entities (Dehaene, 1997, specially
} 
resort, to a reproducible counting (Thouless, 1998, § 1.1). Etymologically, "discrete", "critical", "criterion", and "discernment" share the same greek root $\kappa \rho i \nu \omega\left(k r^{\prime}\right.$ ino, to judge 4 . However, the boundaries of macroscopic objects, considered both in space and time, remain inevitably blurred. For instance, consider one cherry; through absorption and desorption, a perpetual exchange of matter takes place at small scales on the skin of the cherry, and no one can really identify with a precision of one second the time when this cherry has appeared from a blossom or destroyed by natural deterioration. 5 . This ambiguity was known from antiquity and supply the sorites paradox (what is the minimum number of grains in a heap of sand?) - and the paradox of the ship of Theseus (Plutarch asks if, after decades of restauration, once her last plank has been replaced, the ship remains the same Theseus's ship (Plutarch, 1914, The life of Theseus $\S$ XXIII.1)).

In the second part of the XIxpcentury, experiments allowed to move the debate beyond speculations into the microscopic world. In the same movement, mathematics saw the emergence of a new discipline, topology, where were identified some discrete classifications - first in geometry, then in analysis and algebra - up to continuous invertible transformations (homeomorphisms). The integer numbers upon which the classes of, say, graphs, knots, surfaces, fixed points of a flow, critical points of a real map, are discriminated provide, by essence, a robust quantization; they are topological invariant. To put it in a nutshell, there cannot be "half a hole". The dimension of a space6, its connectedness $\left(\pi_{0}\right)$, its homotopy groups $\left(\pi_{1}, \pi_{2}\right.$ and more generally $\left.\pi_{n}\right)$, the signature of the Hessian of a function at a critical point, are examples of such discrete quantities.

In the beginning of the xxpcentury, quantum physics refuted so masterfully the Leibniz continuity principle (Nature does not make jumps) that it bears this claim in its very name. The general rule - known by Pythagoreans for music-according to which a stable wave in a bounded domain has its frequencies quantized (that is, function of integer numbers) now applied at a fundamental level to the Schrödinger waves, which described the states of elementary particles, when bounded. The discrete classification of chemical elements successfully proposed in 1869 by Mendeleev and the discrete spectral lines corresponding to the Balmer series, the Paschen series, the Lyman series etc. observed in radiation, could be explained within a unifying scheme offered by quantum theory. Eventhough it appears that each atomic energy level has actually a continuous bandwidth, due to the coupling to the electromagnetic field whose scattering states belong to a continuum (the photon has no mass), it is nevertheless quantum theory that confered to "being an integer" a genuine physical property. So far, neither the quantification of the spin nor the quantification of the electric charge, say, can be seen as an approximation of a continuous model and the analogous of the Mendeleiev table in the Standard Model contains a finite number of species of elementary particles - about twenty, non counting as distinct a particle from its associated antiparticle characterised by a handful of quantum number 7. Many attempts have been made for finding a topo-

part I and chap. 9).

${ }^{4}$ The etymology lines of these words can be easily traced back with www.wiktionary.org.

${ }^{5}$ In a contribution to the previous volume of this series (Mouchet, 2015, § 5) I have tried to show how symmetries play a crucial role in the process of abstraction and conceptualisation of a macroscopic object like a cherry.

${ }^{6}$ In fractal geometry, the Hausdorff dimension of a set, which can be irrationnal, is not preserved by a homeomorphism.

${ }^{7}$ The discrete character of some observable properties is all the more strengthened that there exists some superselection rules that make irrelevant any continuous superposition of states differing by some discrete values of this observable. 
logical origin of these quantum numbers, one of the motivation being that topological invariance is much harder to break than symmetry invariance. In condensed matter, topology offers a protection against the effects of impurities or out-of-control perturbations and therefore participates to the reproductibility and the fiability of measurements (Thouless, 1998, § 1.3). The seminal attempt in this direction is Dirac's model of magnetic monopole (Dirac, 1931) whose existence would imply the quantization of the electric charge; however, so far, all the quantizations that have been explained find their root in algebraic properties of the symmetry groups used to build a basis of quantum state: 8 (in the absence of evidence of elementary magnetic monopoles, the fact that the electric charges appear to be always an integer multiple of one unit remains mysterious).

Despite these (temporary?) failures of finding topological rather than algebraic roots for the discrete characteristics of what appears to be elementary particles, the quantum theory of fields offers the possibility of describing some collective effects of those particles whose stability is guaranteed by topological considerations. There exists some configurations of a macroscopic number of degrees of freedom that cannot be created or destroyed by a smooth transformation without passing through an intermediate state having a macroscopic, and therefore redhibitory, energy. Depending on the dimension of the space and of the field describing the model, several such topological defects can be considered (point, lines or surfaces) and have been observed in various condensed states (Chaikin and Lubensky, 1995, chap. 9) including, of course, the quantum fluids where the defects are characterised by quantized numbers that can be interpreted as topological invariants. Vortices, which will be the object of the next two sections, provide typical examples of such topological defects along a line in a 3-dimensional space or localised at one point in a 2-dimensional space for a complex scalar field (or a real bidimensional vector field). Under certain circumstances, these collective effects share many properties with the so-called ordinary particles. Since, theoretically, the distinction between the quasi-particles and particles appears, after all, to be just a matter of convention on the choice of the vacuum and of the particles that are considered to be elementary, one may have the secret hope that at a more fundamental level, having the Standard Model as an effective theory, topology shall have the next, but presumably not the last, word.

\section{Classical vortices}

... when I first opened my eyes upon the wonders of the whirlpool... Edgar Allan Poe. A Descent into the Maelström (1841).

\subsection{How vortices participate to the dynamics of the world according to Leonardo and Descartes}

By strong contrast with the still, rather mineral, backgrounds of his paintings, Leonardo da Vinci's interest for the dynamics of water is manifest in his drawings and writtings all along his life. Vortices in water, in air, and even in blood (Pasipoularides, 2010,

\footnotetext{
${ }^{8}$ Topological properties of these Lie groups, obviously their dimensions but also their compactness, their connectedness and their simple connectivity, do play a role but the algebraic commutation relations of their generators remain the main characteristics, which are local ones, that allow to build the irreducible representations defining the one-particle states.
} 
$\S 3.3)$, were a recurrent source of fascination for him 9 . Not only as esthetical motifs (fig. 1), not only because of their crucial role for understanding hydraulics and fly, not only because they inspired him fear as a disordered manifestation of flooding or deluge, but also because they provided a central key for his global conception of the dynamics of the world: l'acqua, vitale omore della terreste macchina, mediante il suo natural calore si move. (water, vital humour of the terrestrial machine, moves by means of its natural heat 10 (Arasse, 1997, Chap. Une science en mouvement).
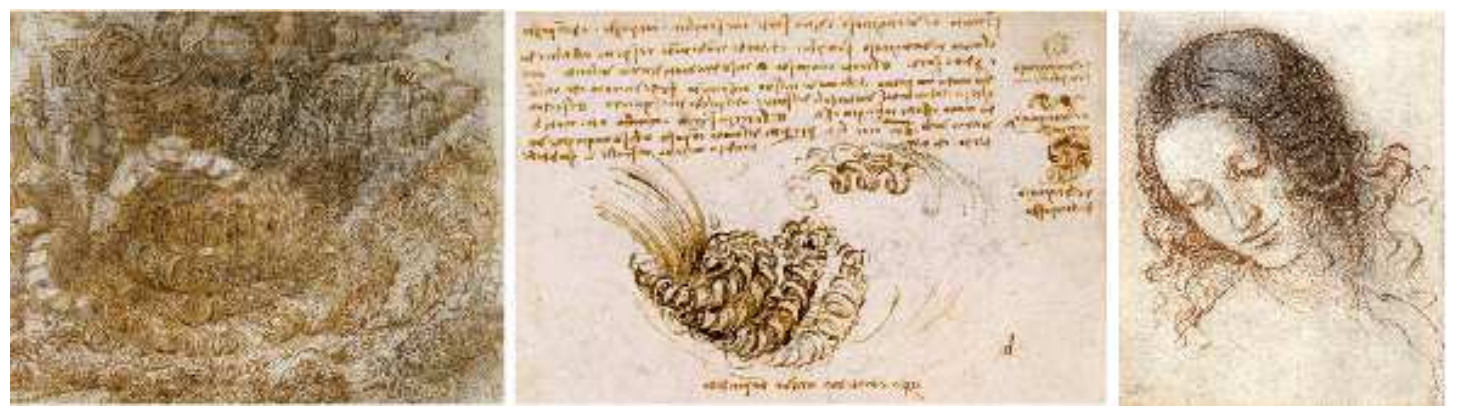

Figure 1: Left) folio w12380, A Déluge, 1517-18. Center) folio w12663r, Studies of flowing water, $\sim 1510-13$. Right) folio w12518, Head of Leda, $1504-1506$. Wikimedia Foundation. On the folio w12579r Leonardo has drawn four studies of vortex alleys formed in water behind a parallelepipedic obstacle and writes Nota il moto del livello dell'acqua, il quale fa a uso de' capelli, che hanno due moti, de' quali l'uno attende al peso del vello, l'altro al liniamento delle volte: cosi l'acqua ha le sue volte revertiginose, delle quali una parte attende al impeto del corso principale, l'altra attende al moto incidente e refresso. (Observe the movement of the surface of water, like hair which has two movements, one due to its weight, the other following the lines of the curls: thus water has whirling eddies, in part following the impetus of the main stream, in part following the incidental and reversed motion, folio w12579r, trad. AM).

More than a century later, most probably without any influence from Leonardo, Descartes put the vortices in the very core of his cosmological model. Rejecting the atomist concept of a vacuum separating matter (Descartes, 1644, part II, 16th principle), he writes

[...] putandum est, non tantum Solis \& Fixarum, sed totius etiam coeli materiam fluidam esse.

([...] we think that not only the matter of the Sun and of the Fixed Stars is fluid but also is the matter of all the sky, trad. AM)

\section{(Descartes, 1644, § III.24 p. 79)}

Being aware of the proper rotation of the Sun (it takes 26 days for the sunspots to complete one turn (Descartes, 1644, § III.32 p. 83)) and of the different orbital period of the planets, he pursues further the hydrodynamical analogy

\footnotetext{
'Gombrich (1969) saw in the exuberance of the terms used by Leonardo and in the profusion of his drawings an attempt to classify the vortices, a line of investigations he kept in mind throughout his life.

${ }^{10}$ Folio H95r, whose facsimile and transcription can be found on www.leonardodigitale.com.
} 
[...] putemus totam materiam coeli in qua Planetae versantur, in modum cuiusdam vorticis, in cuius centro est Sol, assidue gyrare, ac eius partes Soli viciniores celerius moveri quam remotiores [...]

([...] we think that all the matter of the sky, in which the Planets turn, rotates like a vortex with the Sun at its center; that the parts near the Sun move faster than the remote ones [...], trad. AM)

(Descartes, 1644, $\S$ III.30 pp. 81-82)

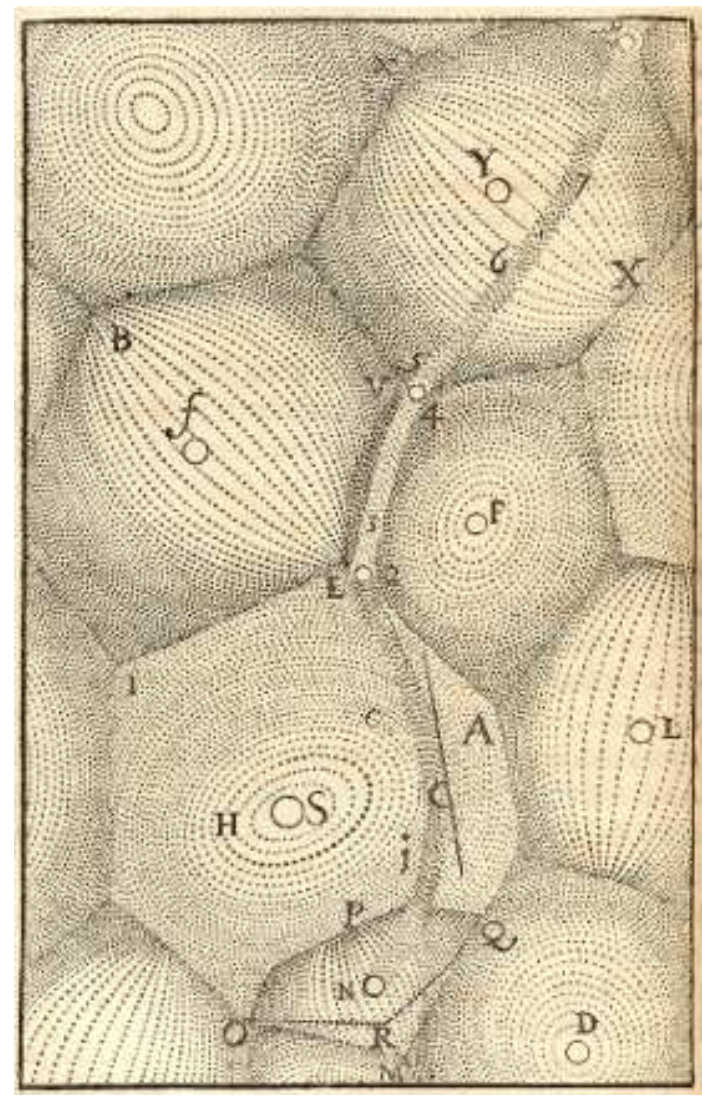

Figure 2: Descartes'vortex-based cosmology. Each star denotes by F, D, etc. is at the center of a vortex. The Sun is denoted by S (Descartes, 1644, § III.23 p. 78).

Descartes'model was overuled by Newton's theory planetary motion but, somehow, in contemporary astrophysics, vortices are still present - in a complete different way, of course, from Descartes' - and triggered by gravitational field acting through the interstellar vacuum: one may think of protoplanetary accretion disks (turbulence plays a crucial role, in particular in the initial molecular cloud for explaining the scattered births of stars) and, at much larger scales, of galaxies, cosmic whirlpools spinning around a giant black hole.

\subsection{Accompanying the birth of topology in the xixpcentury}

His study of the physical properties of organ pipes led Helmholtz to scrutinize the motion of the air near sharp obstacles and the influence of viscosity. The memoir he published in 
German in 1858 on the subject had a decisive influence on the physicists of the Scottish school including Maxwell, Rankine, Tait and Thomson (who was ennobled in 1892 as Lord Kelvin), all the more that Tait translated it into English in 1867 under the title On the integrals of the hydrodynamical equations, which express vortex-motion (Helmholtz, 1867). Inspired by the parallel between mechanics of continuous media and electromagnetism (Darrigol, 2005, chap. 4), Helmholtz showed that, given a field of velocities $\vec{v}$, its curl, the vorticity field,

$$
\vec{\omega}=\overrightarrow{\operatorname{curl}} \vec{v}
$$

is a vector field proportional to the local rotation vector of the fluid. Helmholtz introduced the notion of vortex line (a curve tangent to $\vec{\omega}$ at each of its points) and vortex filament/tube (a bunch of vortex lines) and proved that during its evolution each vortex line follows the motion of the fluid. The dynamical equation of $\vec{\omega}$ allowed him to study precisely the dynamics of straight (fig. 3) and circular vortex tubes (fig. (4). A thin vortex ring whose radius $R$ is much larger than the radius of the cross section of the tube that defines it moves perpendicularly to its plane with the velocity of its center increasing with $R_{11}^{11}$. Based on the similar mathematical problem arose in electrostatics and magnetostatics, Helmholtz understood that the topology of the irrotational part of the flow was essential to determine globally the velocity potential $\alpha$ : in the set of the points $P$ where $\vec{\omega}(P)=0$ one can always locally define a scalar field $\alpha$ such that

$$
\vec{v}=\overrightarrow{\operatorname{grad}} \alpha
$$

but

If we consider [a vortex-filament] as always reentrant either within or without the fluid, the space for which [equation (2)] holds is complexly connected, since it remains single if we conceive surfaces of separation through it, each of which is completely bounded by a vortex-filament. In such complexly connected spaces a function $[\alpha]$ which satisfies the above equation can have more than one value; and it must be so if it represents currents reentering, since the velocity of the fluid outside the vortex-filaments are proportional to the differential coefficients of $[\alpha]$, and therefore the motion of the fluid must correspond to ever increasing values of $[\alpha]$. If the current returns to itself, we come again to a point where it formely was, and find there a second greater value of $[\alpha]$. Since this may occur indefinitely, there must be for every point of such a complexly-connected space an infinite number of distinct values of $[\alpha]$ differing by equal quantities like those of $\tan ^{-1} \frac{x}{y}$, which is such a many-valued function [...].

$$
\text { (Helmholtz, 1867, § 3, translation by Tait). }
$$

The topological properties of vortices can also be understood from what is now known as Kelvin's circulation theorem (Thomson, 1869, §59d) which unified Helmholtz results: in an inviscid (no viscosity), barotropic (its density is a function of pressure only) fluid,

\footnotetext{
${ }^{11}$ In particular, when two rings moving along the same direction get close, the flow created around the leading ring tends to shrink the following one which, conversely, generates a flow that tends to expend the ring ahead. Therefore the leading ring slows down while the second one is sped up until it overtakes the former by passing through it, and the role of the rings are exchanged. This tango, predicted and observed by Helmholtz, is described in the end of his 1858 memoir.
} 

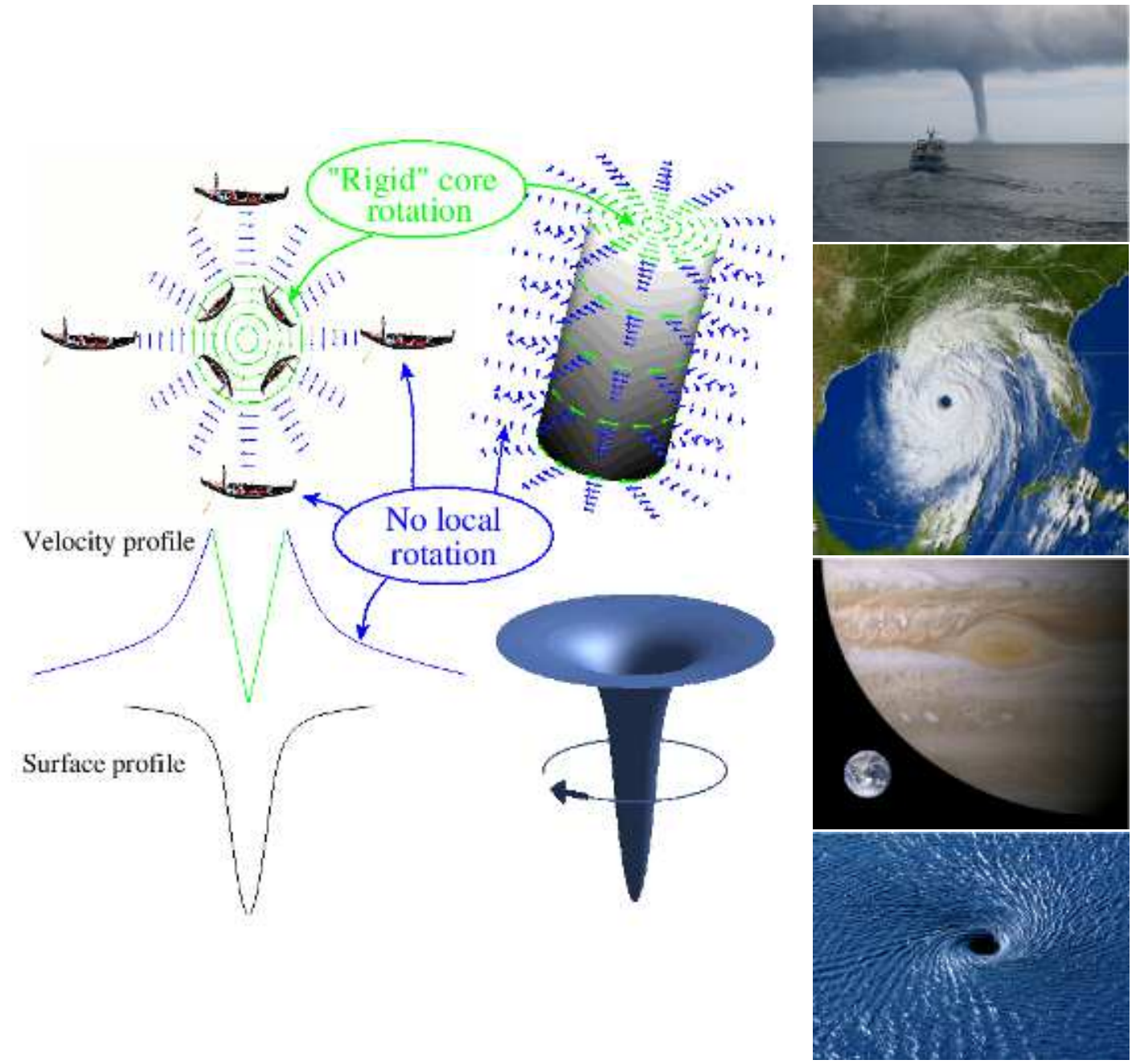

Figure 3: The same year Helmholtz published his seminal memoir, the simplest model of vortex was explicitely proposed by Rankine in (Rankine, 1858, §§ 629-633) who refers to some previous theoretical analysis made by the engineer and physicist James Thomson, inventor of the vortex wheel and brother of William. The vorticity (11) is constant and uniform inside a cylinder - in green, where the fluid rotates as a solid core and the particles rotate around themselves (the axis of the gondola rotates) - and zero outsidein blue, where the fluid particles do not rotate around themselves (the axis of the gondola keeps the same direction). When coming closer to the axis of the vortex, the velocity increases with the inverse of the distance outside the cylindrical core (and then producing a spiral-like shape) and then linearly gets to zero inside the core. In a more or less realistic way, Rankine's vortex models hurricanes, tornados or simply water going down a plughole (image credit: wikipedia, NOAA).

the flux of the vorticity

$$
\Gamma=\int_{\mathscr{S}} \vec{\omega} \cdot \mathrm{d} \vec{S}=\int_{\partial \mathscr{S}} \vec{v} \cdot \mathrm{d} \vec{l}
$$

through a surface $\mathscr{S}$ following the motion of the fluid - or equivalently, according to Stokes' theorem, the circulation of the velocity through the boundary $\partial \mathscr{S}$ of $\mathscr{S}$-is 

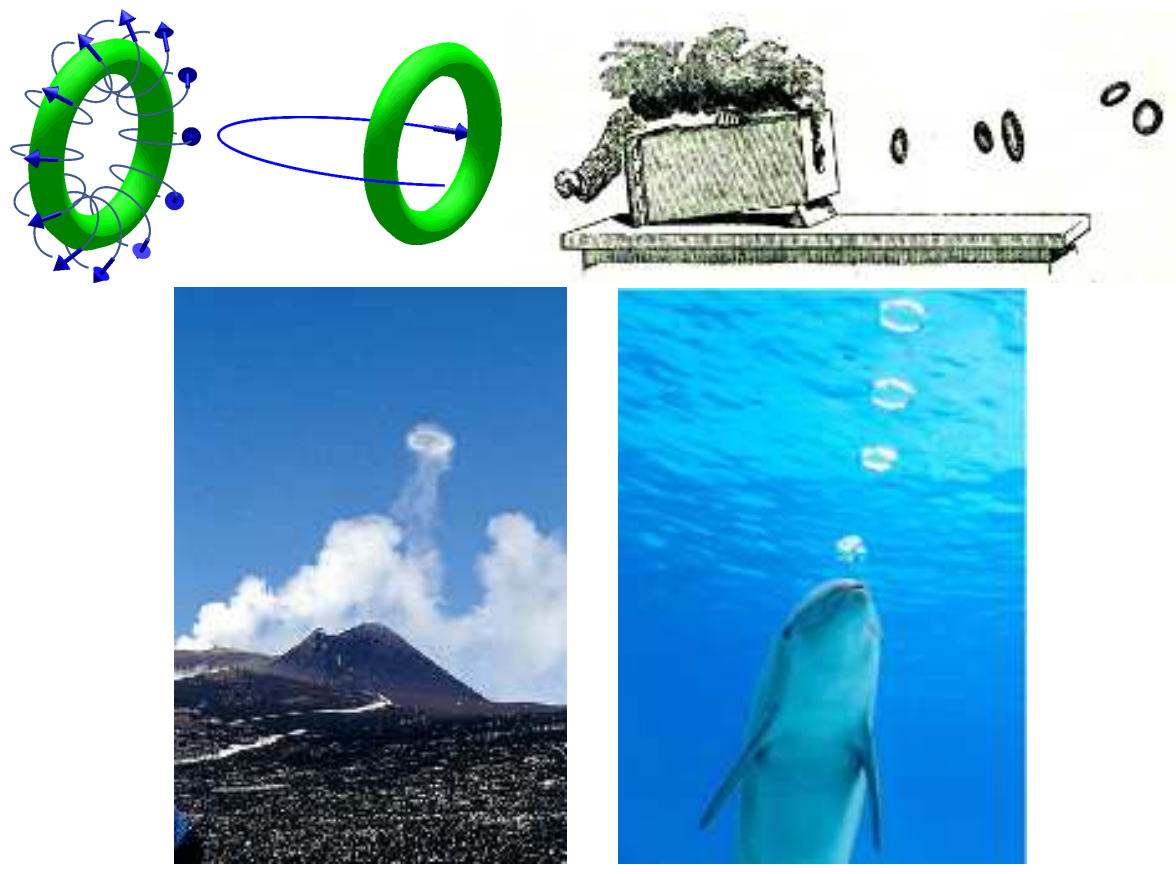

Figure 4: When some vortex lines are bended into a circular tube (in green), each portion of the ring is dragged in the same direction by the fluid whose motion is induced by the other parts of the ring. As a result, a global translation perpendicular to the ring occurs. Helmholtz'study of the dynamics of the rings and the tango played by two interacting rings moving in the same direction, see footnote $11 \mathrm{p}$. 6. can be visualised with Tait's smoke box (upper right taken from (Tait, 1884, p. 292)). In exceptional circumstances vapour rings can be naturally produced by vulcanos (the lower left photograph is taken at Etna by the vulcanologist Boris Behncke, INVG-Osservatorio Etneo). Dolfins and whales are able to produce vortex rings in water (lower right from youtube).

constant. As a consequence, we recover Helmholtz statement that the non simple connectedness of the space filled by the irrotational part of the flow, i.e. the complementary of the vortex tubes, prevents the existence of a continuous globally-defined $\alpha$ and the circulation $\Gamma$ depends on the homotopy class of the loop $\mathscr{C}=\partial \mathscr{S}$. In such an ideal fluid, the vortex lines were therefore topologically stable and Thomson's saw in this stability a key for the description of atomic properties without referring to the corpuscular image inheritated from the atomists of antiquity, which was a too suspicious philosophy for Victorian times (Kragh, 2002, $\S \S 2$ and 9) 12. Since vortex tubes cannot cross transversaly 13 otherwise it is easy to find a $\mathscr{C}$ that does not satisfy Kelvin's theorem, the knot formed by a closed vortex tube and the intertwinning between several such closed loop

\footnotetext{
${ }^{12}$ Some smoothness into the atom had already been introduced by Rankine in 1851 with his hypothesis of molecular vortices according to which "each atom of matter consists of a nucleus or central point enveloped by an elastic atmosphere, which is retained in its position by attractive forces, and that the elasticity due to heat arises from the centrifugal force of those atmospheres, revolving or oscillating about their nuclei or central points" (Rankine, 1851, § 2). It is worth noting that Rankine acknowledges the pertinence of William Thomson's comments on the first version of this 1851's proposal.

${ }^{13}$ But, it seems that neither Helmholtz nor Thomson have considered the possibility of a longitudinal merging of vortex tubes, forming a trousers-like shape (Fuentes, 2007, in particular fig. 6).
} 
remain topologically invariant.

The absolute permanence of the rotation, and the unchangeable relation you have proved between it and the portion of the fluid once acquiring such motion in a perfect fluid, shows that if there is a perfect fluid all through space, constituting the substance of all matter, a vortex-ring would be as permanent as the solid hard atoms assumed by Lucretius and his followers (and predecessors) to account for the permanent properties of bodies (as gold, lead, etc.) and the differences of their characters. Thus, if two vortexrings were once created in a perfect fluid, passing through one another like links of a chain, they never could come into collision, or break one another, they would form an indestructible atom; every variety of combinations might exist.

Thomson to Helmholtz, January 22, 1867, quoted by (Kragh, 2002, p. 38).

The theory of the vortex atoms offered to Thomson the possibility of making concrete his long-standing intuition of a continuous conception of the world, as he had confessed it to Stokes

Now I think hydrodynamics is to be the root of all physical science, and is at present second to none in the beauty of mathematics.

Thomson to Stokes, December, 20, 1857, quoted in (Kragh, 2002, p. 35)

Despite the physical failure of Thomson's ambitious aim (Silliman, 1963; Epple, 1998; Kragh, 2002)14, the identification of topological invariants on knots, upon which the classification of atoms and molecules would have been based, and the classification of the knots by Tait (see Fig. 5 for instance) remains a groundbreaking mathematical work, with direct repercussions in contemporary topology.

One of the Thomson's greatest hopes, while spectroscopy was gathering more and more precise data, was to explain the origin of the discrete spectral lines with " [...] one or more fundamental periods of vibration, as has a stringed instrument of one or more strings [...]" (Thomson, 1867, p. 96). One cannot prevent to find an echo of this motivation in modern string theory where "each particle is identified as a particular vibrational mode of an elementary microscopic string" (Zwiebach, 2004, § 1.2) - see also (Cappelli et al., 2012, in particular § 19). Not without malice, Kragh (2002) was perfectly right to qualify Thomson's dream as a "Victorian theory of everything".

\section{Quantum vortices}

\subsection{Topological origin of quantized flux in quantum fluids}

Unlike what occurs in classical fluids where viscosity eventually make the vortices smoothly vanish, quantum fluids provide a state of matter, much more similar to ideal fluids, where

\footnotetext{
${ }^{14}$ As far as classical hydrodynamics is concerned, some progress have been made in the xxpcentury with, for instance, the identification of new integrals of motion constructed from topological invariants like the Calugareanu helicity (Moffatt, 2008) ; experimentally some not trivial knotted vortices could be produced only recently (Kleckner and Irvine, 2013).
} 


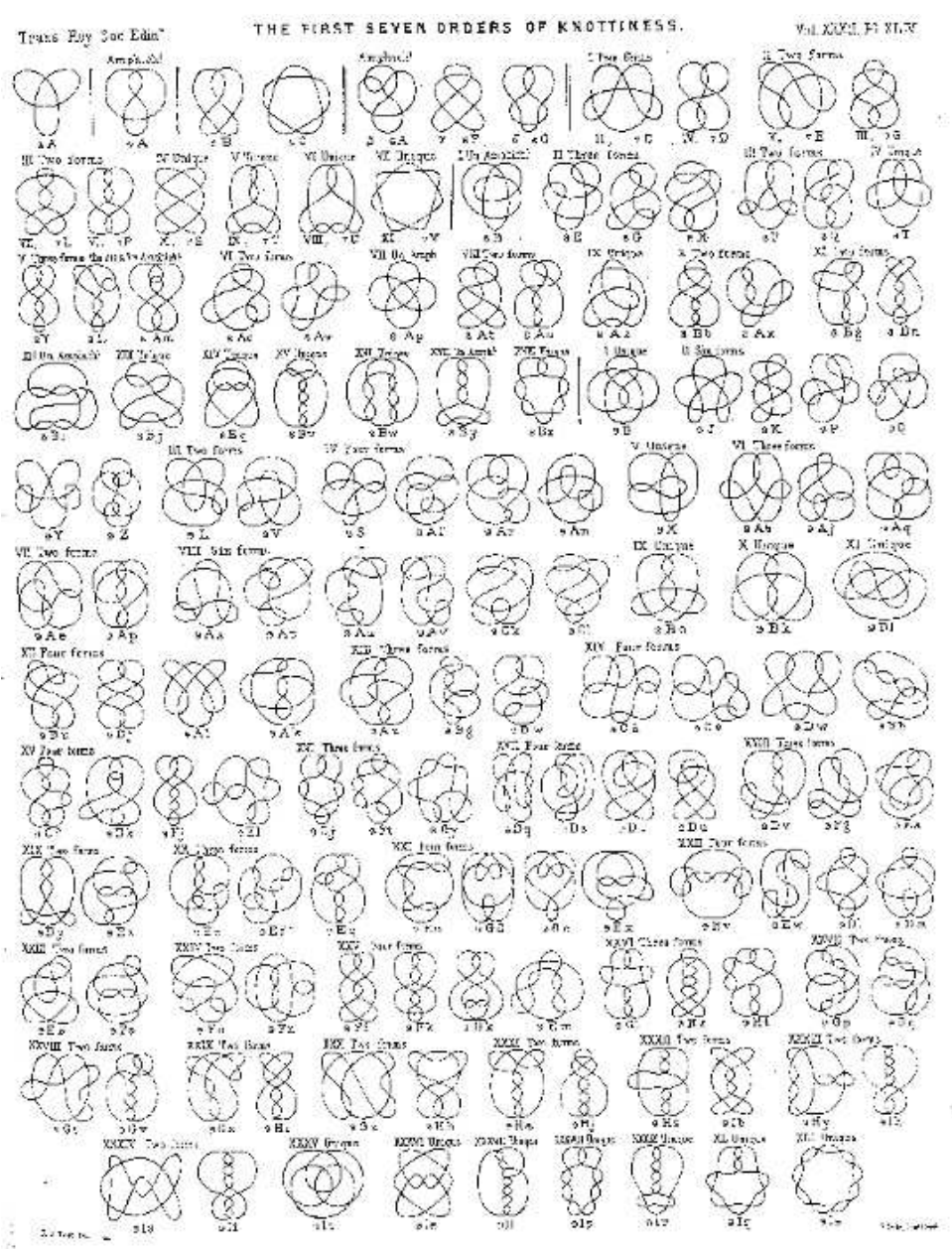

Figure 5: List of knots up to the seventh order established by Tait (1884, Plate XLIV between p. $338 \& 339)$.

vortices are strongly protected from dissipative processes. Indeed, at low temperature, particles can condensate into a collective quantum state where transport can be dissipationless: this is one of the main characteristics of superconductivity (discovered in solid mercury below $4 \mathrm{~K}$ by Onnes in 1911), superfluidity (discovered in liquid Helium-4 below 2K by Kapitsa and Allen \& Misener in 1938), and Bose-Einstein condensate of atoms (discovered for rubidium below $170 \mathrm{nK}$ by Cornell \& Wieman and Ketterle in $1995 \sqrt{15}$. There is a second reason, of topological origin, that reinforces the stability of the vortices in quantum fluids: the scalar field $\alpha$ whose gradient is proportional to the current is not a simple mathematical intermediate as in the classical case (see (2)) but acquires the more physical status of being a phase (an angle) that may be measured in interference experiments like in the Aharonov-Bohm effect. As a consequence, on any

\footnotetext{
${ }^{15}$ One can find many textbooks at different levels and more or less specialised to one type of quantum fluids. To get an introductory bird's-eye view on quantum fluids and other matters in relation to statistical physics, my personal taste go to (Chaikin and Lubensky, 1995), (Huang, 1987) and the particularly sound, concise, and pedagogical (Sator and Pavloff, 2016) (in French).
} 
closed loop $\mathscr{C}$, the circulation $\Gamma$ given by (3) has to be an integer multiple of $2 \pi$ :

$$
w[\mathscr{C}] \stackrel{\text { def }}{=} \frac{1}{2 \pi} \int_{\mathscr{C}} \overrightarrow{\operatorname{grad}} \alpha \cdot \mathrm{d} \vec{l} \in \mathbb{Z} .
$$

Since smooth transformations cannot provoque discrete jumps, $w$ is therefore topologically protected. In other words, the flux of $\overrightarrow{\operatorname{curl}} \vec{v}$ - which keeps its physical interpretation of being a vorticity in superfluids as well as in Bose-Einstein condensates of atoms, whereas it represents a magnetic field in superconductor 16 is quantized and naturally leads to elementary vortices carrying a unit flux quantum. As a matter of fact, the quantum fluid state is described by a complex field $\psi=|\psi| \mathrm{e}^{\mathrm{i} \alpha}$ (the order parameter) and $w[\mathscr{C}] \neq 0$ denotes a singularity of the order parameter on any surface $\mathscr{S}$ whose boundary is $\mathscr{C}$. Vortices constitute a particular case of what is generally called a topological defect whose dimension depends on the dimension of the order parameter and on the dimension of the space. At microscopic scales, very much like in the Rankine model, the vortex is made of a core outside which $\overrightarrow{\operatorname{curl}} \vec{v}=0$; the vorticity/magnetic lines are trapped inside the core where the density of the superfluid $|\psi|^{2}$ tends to zero at its center. Not only, these vortices have been observed in all the three types of superfluids mentioned above but also the triangular lattice they form to minimize the (free) energy due to an effective repulsion between them first predicted by Abrikosov (1957), see fig [6). When the fluctuations of $|\psi|$ in space and time are negligible, notably at sufficiently low temperatures, the quantum fluid is essentially described by the phase $\mathrm{e}^{\mathrm{i} \alpha}$ or equivalently by a bidimensional vector of unit norm oriented at angle $\alpha$ with respect to a given direction (fig. 7).

\subsection{The XY-model}

The latter picture is known as the XY-model, which is also relevant for some classical liquid crystals or for systems of classical spins (Chaikin and Lubensky, 1995, chap. 6). At macroscopic scales, some collective effects of such model are not very sensitive to the details of the interaction nor to the geometry of the elementary cell in the case of a lattice but depend crucially on the dimension $d$ of the space of positions (the number of components of $\vec{r}$ ).

Typically, the energy of the system increases when some differences in the orientation $\alpha$ appears; more precisely the energy density contain a term proportional to $(\overrightarrow{\operatorname{grad}} \alpha)^{2}$. It is not affected by a homogenous rotation of all the spins,

$$
\alpha(\vec{r}) \mapsto \alpha(\vec{r})+\alpha_{0},
$$

where the angle $\alpha_{0}$ does not depend on $\vec{r}$. The absolute minimum of the total energy is obtained when all the vectors are aligned, which is the configuration at temperature $T=$ $0 \mathrm{~K}$. When $T>0$, the equilibrium corresponds to more disordered configurations but, for $d=3 \sqrt{17}$, some non-zero average value of $\alpha$ can be maintained up to a critical temperature $T_{\text {critical }}$ beyond which the average value of $\alpha$ is zero (fig. 8). At $d=2$, on the contrary, the correlations between fluctuations never decrease sufficiently rapidly at

\footnotetext{
${ }^{16}$ Compare (1) with the relation $\vec{B}=\overrightarrow{\operatorname{curl}} \vec{A}$ between the (gauge) vector potential $\vec{A}$ and the magnetic field $\vec{B}$.

${ }^{17}$ Surprisingly, as far as the computations are concerned, the integer nature of $d$ becomes secondary and one can formally consider $d$ as continuous. The condition for an order/disorder phase transition at $T_{\text {critical }}>0$ to exist is $d>2$.
} 

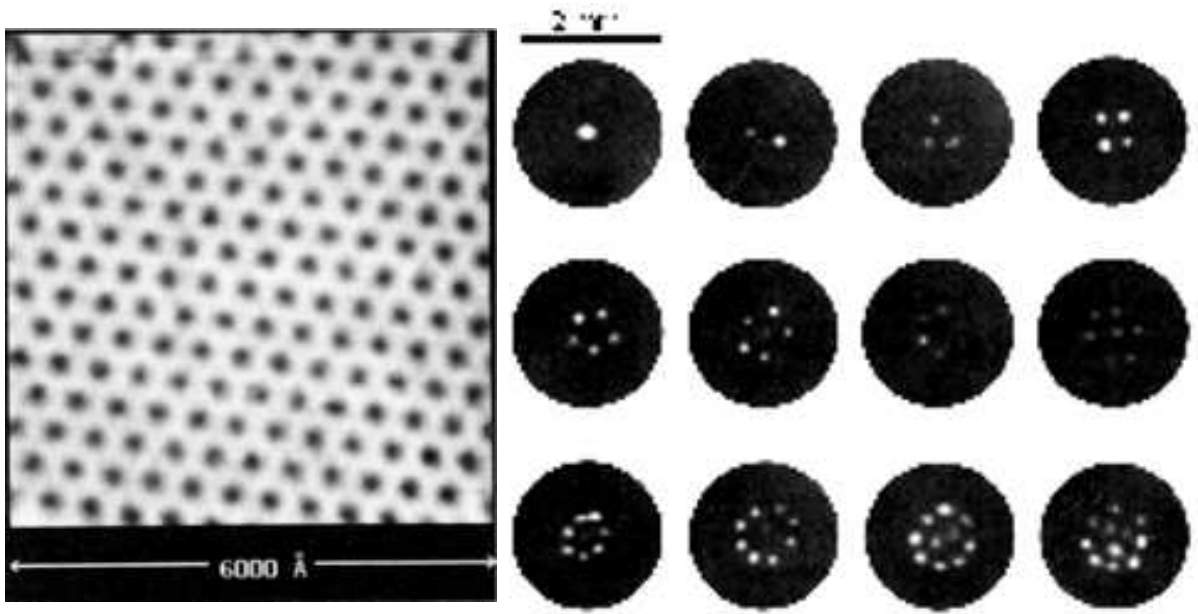

(a)
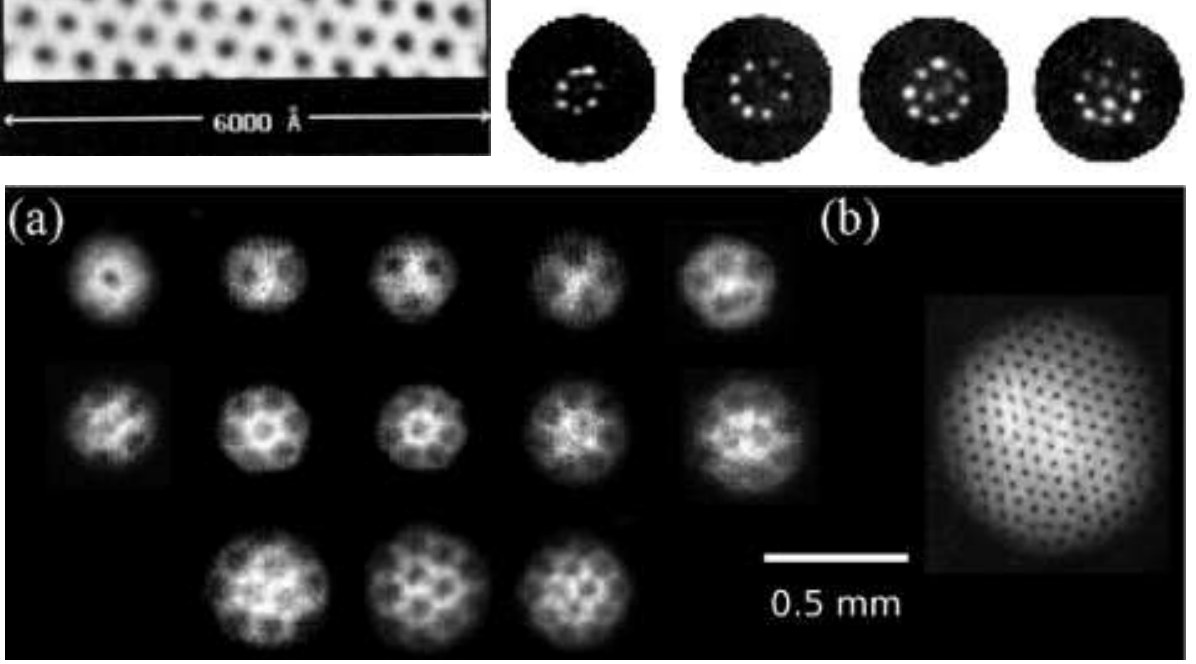

Figure 6: Up left) Abrikosov lattice of vortices in a superconductor (Hess et al., 1989, fig. 2). Up right) Vortices in superfluid helium (Yarmchuk et al., 1979, fig. 2). Below) Vortices in a rotating Bose-Einstein condensate obtained by a) Dalibard's group (Madison et al. 2000, fig. 1 \& Chevy and Dalibard 2006, fig. 4); b) Ketterle's group (Raman et al., 2001, fig. 4c). (C)European Physical Society and American Physical Society.

large distances and the average value of $\alpha$ is zero as soon as $T>0$. However one can still identify, at some finite temperature $T_{\text {critical }}>0$, a qualitative change of behaviour in the correlation lengths, from a power-law decay at large distances to an exponential decay and this phase transition has observable repercussions, notably in superfluids helium films (Bishop and Reppy, 1978). The theoretical description of what appeared to be a new kind of phase transition, now known as topological phase transitions, was proposed by Kosterlitz and Thouless (1972) who showed that vortices were a cornerstone of the scheme.

As soon as their first papers, Kosterlitz and Thouless, talked about "topological order" because they were perfectly aware that this type of phase transition, unlike all the phase transitions known at the time of their publication, relies on topology rather than on symmetry (breaking). As we have seen above on eq. (4), each vortex (now a topological defect of one dimension) is characterised by an integer, called the topological index of the vortex which can be reinterpreted using the concepts introduced by Poincaré in a series of papers that can be considered as the foundations of topology as a fully autonomous 
research discipline (Epple, 1998, § 4). Any direction far away a topological defect of dimension $f$ in a space of dimension $d$ is represented by an element of the rotation group in $n=d-f-1$ dimensions, in other words such a defect can completely enclosed by a $n$-dimensional sphere $S_{n}$. In $d=3$ dimensions a wall (a surface of dimension $f=2$ ) cannot be enclosed $(n=0)$, a vortex-line $(f=1)$ can be enclosed by a circle $(n=1)$, a point $(f=0)$ can be enclosed by a $n=2$-sphere. In $d=2$ dimensions a wall (a line of dimension $f=1)$ cannot be enclosed $(n=0)$ and a point can be enclosed by a circle $(n=1)$. To each direction one can associate the value of the order parameter and therefore to each defect one gets a map from $S_{n}$ to $\mathscr{P}$ where $\mathscr{P}$ denotes the space to which the order parameter belongs. In the examples above $\mathscr{P}$ is just the set $S_{1}$ of the angles $\alpha$ but much more different situations may be encountered. For $n=1$, any loop $\mathscr{C}$ around a given point maps on a closed path $\mathscr{C}^{\prime}$ in $\mathscr{P}=S_{1}$ and the topological index $w$ of the point is just the winding number of $\mathscr{C}^{\prime}$ (figs. 9 and 10).

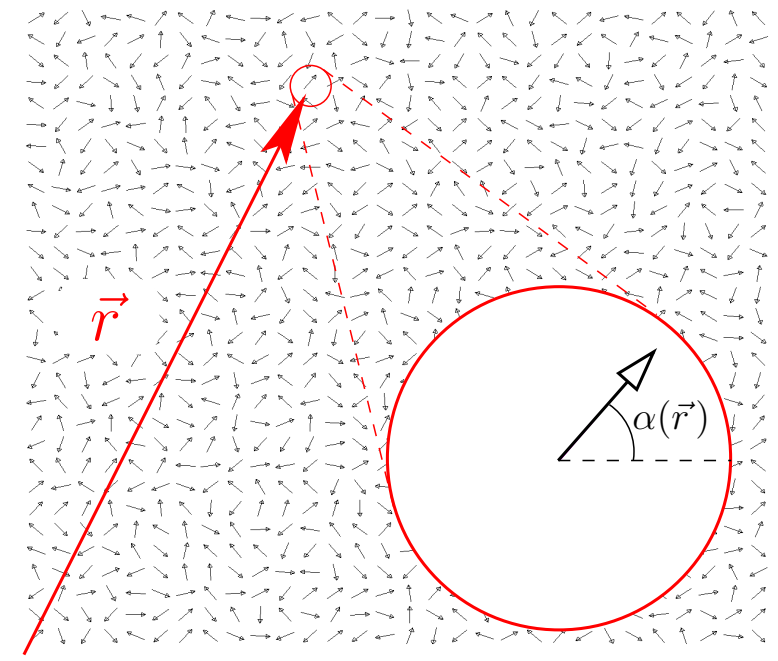

Figure 7: The XY-model describes an interacting bidimensional vector field of constant and uniform norm. On a continuous space or on a lattice, the direction of the field at point $\vec{r}$ is given by one angle $\alpha(\vec{r})$. 


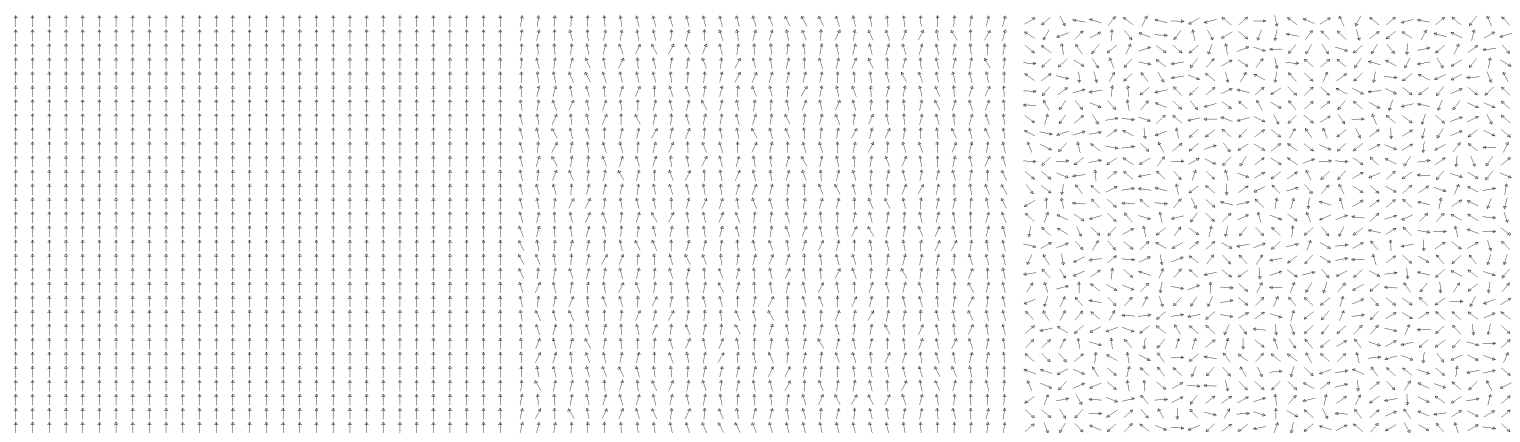

Figure 8: In three dimensions the XY-model presents order/disorder phase transition, very similar to the familiar solid/liquid phase transition. Below a critical temperature $T_{\text {critical }}>0$ some order is maintained throughout the system at macroscopic lengths (middle picture) with the perfect order obtained at $T=0 \mathrm{~K}$ (left picture). Above $T_{\text {critical }}$, the average orientation is zero and no more order at large scales can be identified (right picture).

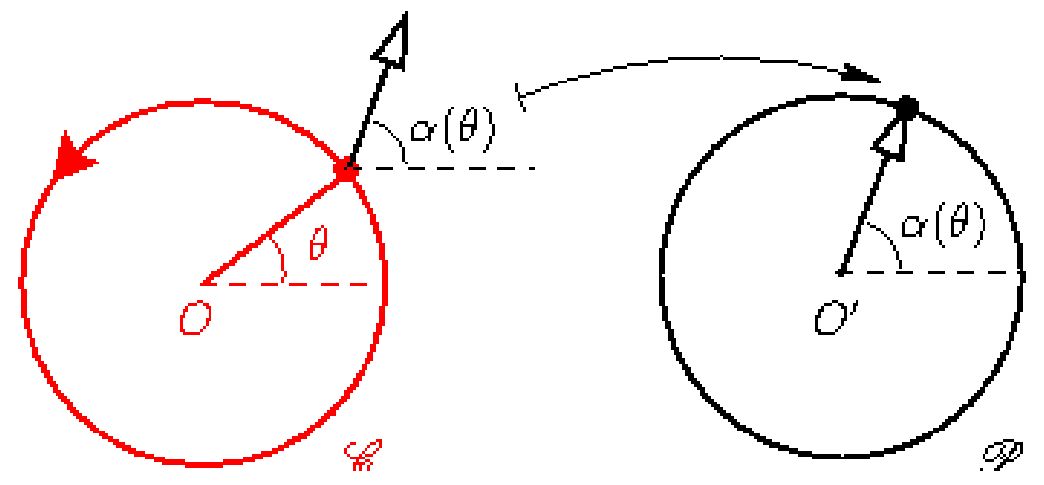

Figure 9: For the XY-model, in $d=2$, to each point on a loop $\mathscr{C}$ enclosing any given point 0 (in red, on the left) is associated the direction of the order parameter on the circle $\mathscr{P}$ (in black on the right). 
More generally, the topological invariants are given by the group $\pi_{n}$ of $\mathscr{P}$ (for $n=0$ it provides the connectedness, for $n=1$ it provides the first homotopy group that is the simple connectedness, etc.). A continuous transformation of the configuration cannot modify $w$ at any point and physically it would require a macroscopic amount of energy to change $w$. On the other hand, one configuration having one defect can be deformed continuously at low cost of energy into any other configuration having a defect with the same $w$. In particular, the transformation (5) does not cost any energy at all.

One cannot therefore expect to isolated elementary vortex $(w=1)$ or isolated elementary antivortex $(w=-1)$ to be spontaneously created from a perfect ordered state. Nevertheless, a pair of vortex-antivortex is affordable when $T>0$ (fig. 11). The continuous creation (or annihilation) of such a pair can be understood by considering the appearance of a fold on a drapery (back to Leonardo again?). One may intuitively see that this is a generic process, stable with respect to smooth transformations, that describes the creation or the annihilation of a pair of maximal-minimal points on a smooth function (fig. 12) or, equivalently, the creation or annihilation of intersection points

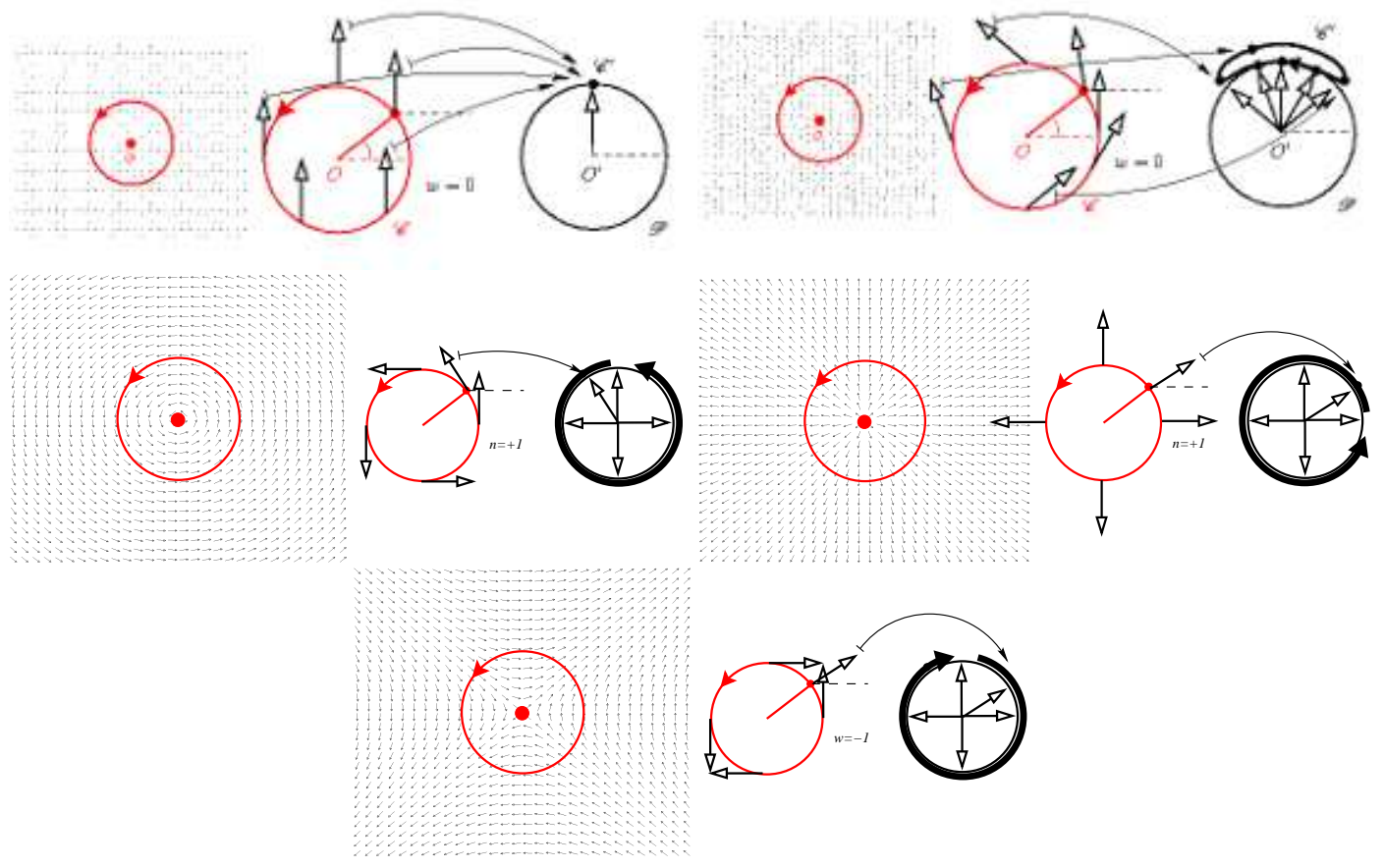

Figure 10: In the XY-model, the topological index (44) of a point $O$ is the winding number of the curve $\mathscr{C}^{\prime}$ (thick black line) defined to be the image of a closed loop $\mathscr{C}$ (in red) in the circle $\mathscr{P}$ (thin black circle) that indicate the direction $\alpha$ of the order parameter. A smooth deformation deforms $\mathscr{C}^{\prime}$ but do not change $w$ (we stay in the same homotopy class). The upper row provides two examples having $w=0$ (with, on the left, a uniform order parameter, $\mathscr{C}^{\prime}$ is just a point). The central row provides two elementary vortices $(w=1)$ whose configurations differ from left to right by a rotation (5) with $\alpha_{0}=-\pi / 2$. The lower row provides an example of configuration having an elementary antivortex $(w=-1)$. 


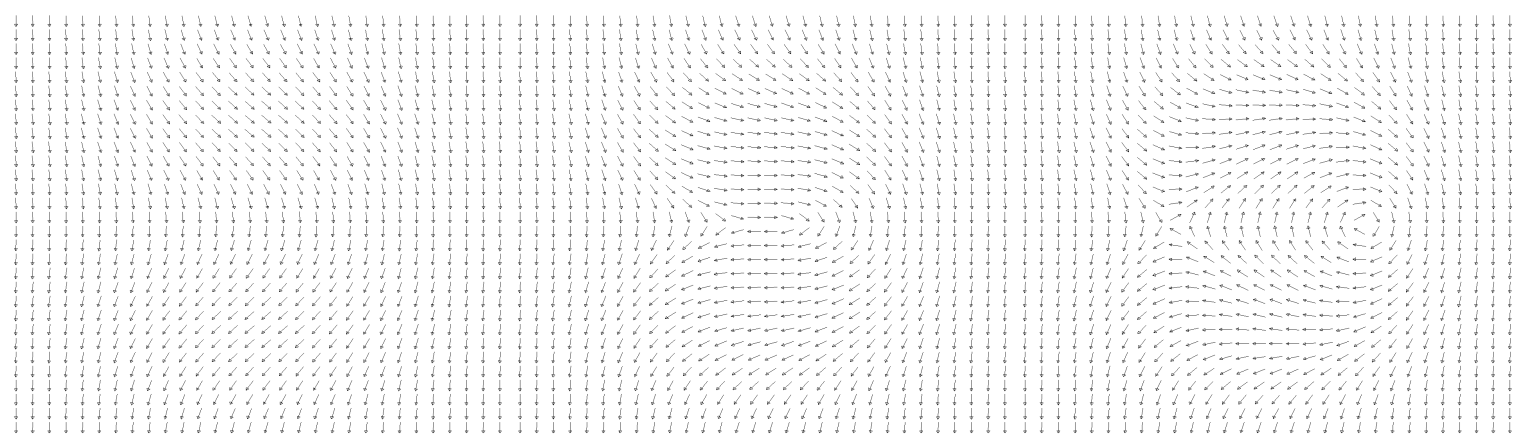

Figure 11: A smooth transformation that does not require a macroscopic amount of energy can make a vortex/antivortex pair to spontaneously appear as a local fluctuation at non-zero temperature. The genericity and the structural stability of this scenario can be understood when considering the appearance of a fold (fig 12).

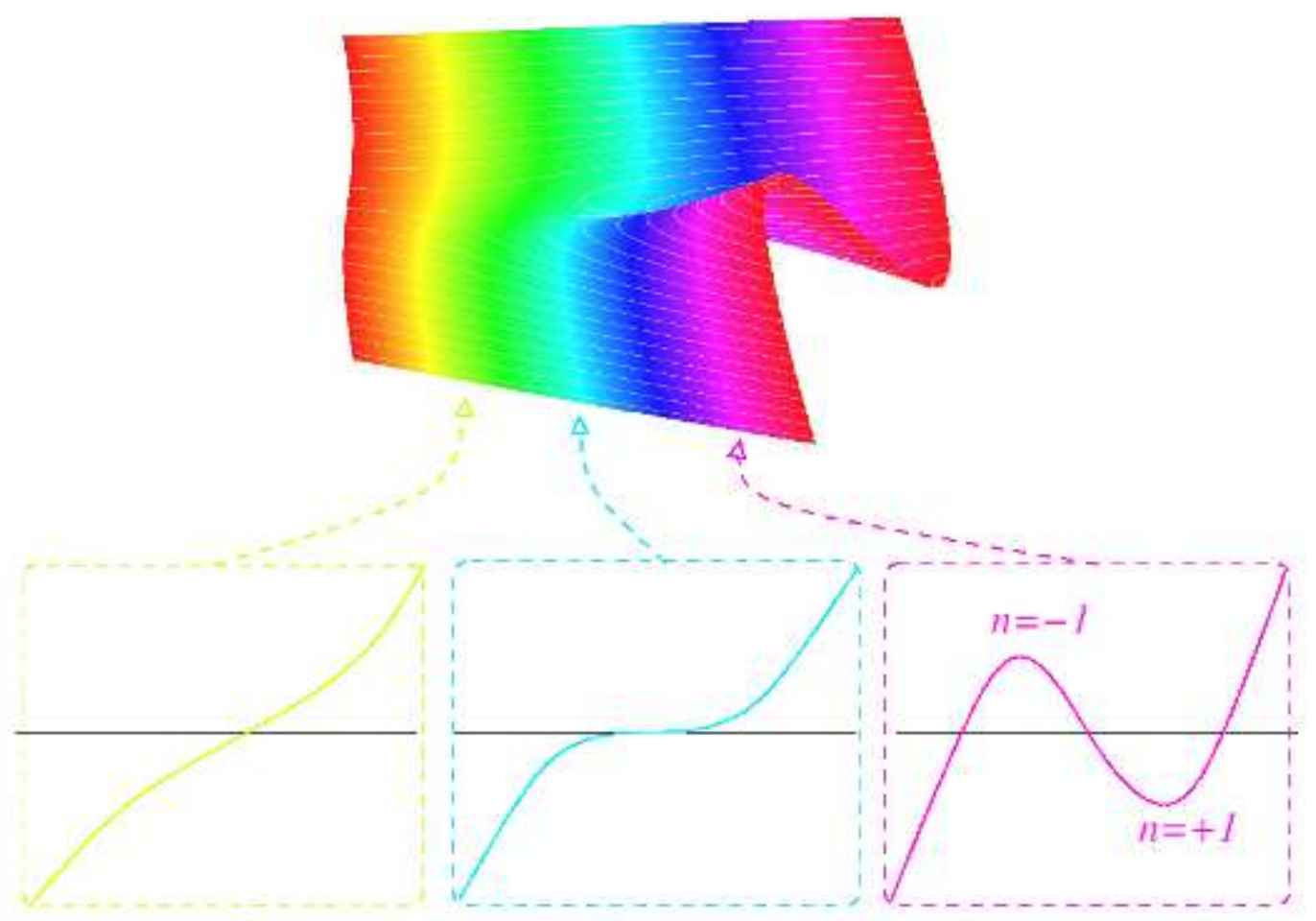

Figure 12: The fold catastrophe is the simplest of the bifurcation scenario. It involves a one real parameter family of functions where two generic critical points (on the right), having opposite second derivatives, merge into a degenerate critical point (central graph) and disappear (on the left). It also represents how generically a non transversal crossing between two tangent curves (in the center) is unfolded from one (on the left) to three (on the right) transversal crossings.

when two curves that cross transversaly are smoothly locally deformed 18 . The topo-

\footnotetext{
${ }^{18}$ Topology is fully at work here and the study of the stability of the critical points of smooth
} 
logical phase transition describes precisely how the creation of an increasing number of vortex-antivortex pairs as the temperature increases eventually lead from a topological order to a state where complete disorder reigns.

\section{Concluding remark}

To come back to issues mentioned in the last paragraph of $\S 1$, in quantum theory, the fundamental elementary particles stem from algebraic symmetry considerations. However, we have some clues (topological defects, solitons, instantons, monopoles, etc.) that topology may offer a complementary ground. The parallel between creation/annihilation of particle-antiparticle pairs and creation/annihilation of vortex-antivortex pairs may be more than a simple analogy.

Thomson/Kelvin's intuition may take an unexpected but relevant form, after all.

Acknowledgement: I am particularly grateful to Pascal Brioist (Centre d'Étude Supérieures de la Renaissance de l'Université de Tours) for his expert advices on Leonardo studies, to Boris Behncke (INVG-Osservatorio Etneo)) for letting me use his photo of the vapour ring created by the Etna (see fig 4) and to Michele Emmer who triggered the subject of this essay for the conference Matematica e Cultura 2017, Imagine Math 6, at Venice.

\section{References}

A. A. Abrikosov. The magnetic properties of superconducting alloys. J. Phys. Chem. Solids, 2(3):199-208, 1957.

D. Arasse. Léonard de Vinci. Hazan, Malakoff, 1997. English translation: Konecky (1998).

V. I. Arnold. Catastrophe Theory. Springer-Verlag, New York, 1984.

J. L. Bell. The Continuous and the Infinitesimal in Mathematics and Philosophy. Polimetrica, Milano, 2006. see also Bell's article with the same title in the Stanford Encyclopedia of Philosophy, plato.stanford.edu.

D. J. Bishop and J. D. Reppy. Study of the superfluid transition in two-dimensional he-4 films. Phys. Rev. Lett., 40(26):1727-1730, 1978.

A. V. Borisov, V. V. Kozlov, I. S. Mamaev, and M. A. Sokolovskiy, editors. IUTAM symposium on Hamiltonian dynamics, vortex structures, turbulence, Dordrecht, 2008. IUTAM bookseries vol. 6, Springer.

A. Cappelli, E. Castellani, F. Colomo, and P. Di Vecchia. The birth of string theory. Cambridge University Press, Cambridge, 2012.

P. M. Chaikin and T. C. Lubensky. Principles of condensed matter physics. Cambridge University Press, Cambridge, 1995.

mappings is the object of catastrophe theory whose greatest achievement is to have classified the generic possible scenarios; the simplest one being precisely the fold catastrophe, depicted in figure 12 (Poston and Stewart, 1978; Arnold, 1984, for a general survey). 
F. Chevy and J. Dalibard. Rotating Bose-Einstein condensates. Europhysicsnews, 37 (1):12-16, 2006. Free access on line at www.europhysicsnews.org.

O. Darrigol. Worlds of Flow. A history of hydrodynamics from the Bernoullis to Prandtl. Oxford University Press, Oxford, 2005.

S. Dehaene. The number sense. How the mind creates mathematics. Oxford University Press, Oxford, 1997.

R. Descartes. Principia philosophice. Ludovicum Elzevirium, Amsterdam, 1644. Latin original edition available on gallica.bnf.fr.

P. A. M. Dirac. Quantised singularities in the electromagnetic field. Proc. Roy. Soc. London Ser. A, 133:60-72, 1931. reproduced in $\S 2.1$. of Thouless (1998).

M. Emmer, M. Abate, and M. Villarreal, editors. Imagine Maths 4. Between culture and mathematics, Venezia e Bologna, 2015. Unione Matematica Italiana. Instituto Veneto di Scienze, Lettere ed arti.

M. Epple. Topology, matter, and space, I: Topological notions in 19th-century natural philosophy. Arch. Hist. Exact Sci., 52:297-392, 1998.

V. Fuentes. On the topology of vortex lines and tubes. J. Fluid Mech., 584:147-156, 2007.

E. H. Gombrich. The form of movement in water and air. In O'Malley (1969), pages 171-204. Reproduced in Gombrich (1976) pp. 39-56.

E. H. Gombrich. The Heritage of Apelles, volume 3 of Studies in the Art of the Renaissance. Phaidon, Oxford, 1976.

H. von Helmholtz. Über Integrale der hydrodynamischen Gleichungen, welche der Wirbelbewegungen entsprechen. J. Reine Angew. Math., 55:22-55, 1858. English translation by P. G. Tait in Helmholtz (1867).

H. von Helmholtz. On the integrals of the hydrodynamical equations, which express vortex-motion. Philos. Mag., supp. to vol. 33 (4th series)(226):485-512, 1867. English translation by P. G. Tait of Helmholtz (1858).

H. F. Hess, R. B. Robinson, R. C. Dynes, J. M. Valles Jr, and J. V. Waszczak. Scanningtunneling-microscope observation of the abrikosov flux lattice and the density of states near and inside a fluxoid. Phys. Rev. Lett., 62(2):214-216, 1989.

K. Huang. Statistical Mechanics. John Wiley and sons, New York, 1987. 2nd edition.

I. M. James, editor. History of topology, Amsterdam, 1999. North-Holland.

D. Kleckner and W. T. M. Irvine. Creation and dynamics of knotted vortices. Nat. Phys., 9:253-258, 2013.

J. M. Kosterlitz and D. J. Thouless. Long range order and metastability in two dimensional solids and superfluids. J. Phys. C, 5:L124-L126, 1972. The detailed version of this work is Kosterlitz and Thouless (1973). 
J. M. Kosterlitz and D. J. Thouless. Ordering, metastability and phase transitions in two-dimensional systems. J. Phys. C, 6:1181-1203, 1973.

H. Kragh. The vortex atom: A Victorian theory of everything. Centaurus, 44:32-114, 2002.

K. W. Madison, F. Chevy, W. Wohlleben, and J. Dalibard. Vortex formation in a stirred bose-einstein condensate. Phys. Rev. Lett., 84(5):806-809, 2000.

K. Moffatt. Vortex dynamics: the legacy of Helmholtz and Kelvin. In Borisov et al. (2008), pages $1-10$.

A. Mouchet. Symmetry: a bridge between nature and culture. In Emmer et al. (2015), pages $235-244$.

M. Nakahara. Geometry, Topology and Physics. Graduate student series in physics. Adam Hilger, Bristol and New-York, 1990.

C. Nash. Topology and physics - a historical essay. In James (1999), pages 359-415, Chap. 12. see also arxiv.org/abs/hep-th/9709135.

C. D. O'Malley, editor. Leonardo's legacy. An international symposium (2-8 May 1966), Berkeley and Los Angeles, 1969. UCLA Center for Medieval and Renaissance Studies (vol.2), University of California Press.

A. Pasipoularides. Heart's vortex. Intracardiac blood flow phenomena. People's Medical Publishing House - USA, Shelton, 2010.

Plutarch. Lives, volume I of Loeb classical library. Harvard University Press, Cambridge, 1914. Bilingual edition commented and translated from the Greek by Bernadotte Perrin.

T. Poston and I. Stewart. Catastrophe theory and its applications. Pitman, London, 1978.

C. Raman, J. R. Abo-Shaeer, J. M. Vogels, K. Xu, and W. Ketterle. Vortex nucleation in a stirred bose-einstein condensate. Phys. Rev. Lett., 87(21):210402, 2001.

W. J. M. Rankine. On the centrifugal theory of elasticity, as applied to gases and vapours. Philos. Mag., supp. to vol. 2 (4th series)(14):509-542, 1851. reproduced in Rankine (1881) § III p. 16-48.

W. J. M. Rankine. A manual of applied mechanics. Professor Rankine's series of practical manuals on civil engineering and mechanics. Richard Griffin and co., London and Glasgow, 1858.

W. J. M. Rankine. Miscellaneous scientific papers. Charles Griffin and compagny, London, 1881.

N. Sator and N. Pavloff. Physique statistique. Vuibert, Paris, 2016. in French.

R. H. Silliman. William thomson: Smoke rings and nineteenth-century atomism. Isis, 54(4):461-474, 1963. 
M. C. Stokes. One and many in pre-socratic philosophy. (Publications of the Center for Hellenic Studies). Harvard University Press, Cambridge, 1971.

P. G. Tait. On knots, Part II. Trans. Roy. Soc. Edin., 32:327-342, 1884.

W. Thomson. On vortex atoms. Proc. Roy. Soc. Edin., 6:94-106, 1867.

W. Thomson. On vortex motion. Trans. Roy. Soc. Edin., 25:217-260, 1869.

D. J. Thouless. Topological Quantum Numbers in Nonrelativistic Physics. World Scientific, Singapore, 1998.

E. J. Yarmchuk, J. V. Gordon, and R. E. Packard. Observation of stationary vortex arrays in rotating superfluid helium. Phys. Rev. Lett., 43(3):214-217, 1979.

B. Zwiebach. A first course in string theory. Cambridge University Press, Cambridge, 2004. 2009 for the 2 nd edition. 